\title{
The Dirichlet Casimir Problem
}

\author{
N. Graham ${ }^{\mathrm{a}}$, R.L. Jaffe ${ }^{\mathrm{b}}$, V. Khemani ${ }^{\mathrm{b}}$, M. Quandt ${ }^{\mathrm{c}}$, O. Schröder ${ }^{\mathrm{b}}$, H. Weigel ${ }^{\mathrm{c}, \mathrm{d}}$

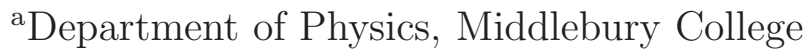 \\ Middlebury, VT 05753 \\ ${ }^{\mathrm{b}}$ Center for Theoretical Physics, Laboratory for Nuclear Science \\ and Department of Physics, Massachusetts Institute of Technology \\ Cambridge, Massachusetts 02139 \\ ${ }^{\mathrm{c}}$ Institute for Theoretical Physics, Tübingen University \\ D-72076 Tübingen, Germany \\ ${ }^{\mathrm{d}}$ Fachbereich Physik, Siegen University \\ D-57068 Siegen, Germany \\ MIT-CTP-3413 UNITU-HEP-14/2003 \\ hep-th/0309130
}

\begin{abstract}
Casimir forces are conventionally computed by analyzing the effects of boundary conditions on a fluctuating quantum field. Although this analysis provides a clean and calculationally tractable idealization, it does not always accurately capture the characteristics of real materials, which cannot constrain the modes of the fluctuating field at all energies. We study the vacuum polarization energy of renormalizable, continuum quantum field theory in the presence of a background field, designed to impose a Dirichlet boundary condition in a particular limit. We show that in two and three space dimensions, as a background field becomes concentrated on the surface on which the Dirichlet boundary condition would eventually hold, the Casimir energy diverges. This result implies that the energy depends in detail on the properties of the material, which are not captured by the idealized boundary conditions. This divergence does not affect the force between rigid bodies, but it does invalidate calculations of Casimir stresses based on idealized boundary conditions.

Keywords: Energy densities, Green's functions, renormalization, Casimir effect

PACS: 11.10.-z, 11.10.Gh, 03.70.+k
\end{abstract}




\section{Introduction}

The development of powerful experimental methods to measure the Casimir force and its potential importance in micromechanical devices have stimulated new interest in the physical principles underlying the Casimir effect [1, 2, 3, 4, The Casimir force was originally discovered by Casimir and Polder in their study of the long range, relativistic tail of the van der Waals force between neutral atoms 5]. Immediately afterwards, Casimir showed that the force between grounded, conducting plates could be understood as a modification of the quantum fluctuations of the electromagnetic fields forced to obey conducting boundary conditions on the plates [6].

The intriguing idea of a force generated by the modification of the zero point energy of a fluctuating quantum field due to the imposition of a boundary condition has generated continuing theoretical interest in generalized "Casimir problems" [7]. The general idea is to specify a) an otherwise free quantum field (scalar, Dirac, gauge, etc.), b) a boundary condition (Dirichlet, confining, conducting, etc.), and c) a geometry (parallel plates, spherical, cylindrical, etc.), and to calculate the forces, pressures, and other potential observables that arise when the field is forced to obey the boundary condition on the surface.

These Casimir problems are idealizations in which the physical interactions between the fluctuating fields and matter have been replaced $a b$ initio by boundary conditions. A real material cannot constrain modes of the field with wavelengths much smaller than the typical length scale of its interactions. The interactions become negligible at wavelengths less than certain physically determined cutoffs. In contrast, a boundary condition constrains all modes. To calculate the Casimir energy it is necessary to sum over the zero point energy of all modes. This sum is highly divergent in the ultraviolet and these divergences depend on the boundary conditions. Subtraction of the vacuum energy in the absence of boundaries removes only the worst divergence (quartic in three space dimensions). We ask whether the Casimir energy and other potential observables can be defined independent of the cutoffs that characterize the actual interactions between the fluctuating fields and the matter. If so, then one can define an abstract Casimir problem, depending on the field, the boundary condition, and the geometry alone. If not, then the Casimir energy is unavoidably entangled with the detailed material physics at hand. We study the simple case of a scalar field, $\phi$, the Dirichlet condition, $\phi=0$, and three geometries: single plate, parallel plates and sphere. We believe this case illustrates general principles that affect all Casimir calculations.

We find that the Casimir energy always depends on the cutoffs and diverges as the cutoffs go to infinity. The divergences in three dimensions are even more severe than the divergences in one and two dimensions described in earlier work 8 . We show that these divergences can be isolated in low-order Feynman diagrams, which can be studied with standard methods of renormalized quantum field theory. It is important to emphasize, however, that the Casimir force between rigid bodies, which is the only thing that has been measured experimentally, 
is cutoff independent and therefore finite in the limit of infinite cutoff. The most important example of an observable that is unavoidably cutoff dependent is the "Casimir pressure" $(-\partial E / \partial A)$, which we study for the case of a sphere.

The method of renormalization in continuum quantum field theory without boundaries (QFT) provides the only physical way to regulate, discuss, and eventually remove divergences. We propose to replace a boundary condition by a renormalizable coupling between the fluctuating field and a non-dynamical background field representing the material. When the background is smooth and the coupling is finite, then renormalization ensures that the zero point energy of the fluctuating field relative to the vacuum is finite. In the limits that the background becomes sharply peaked on a surface and the coupling becomes strong, all modes of the fluctuating field will obey a boundary condition on the surface. If a physical observable like the pressure diverges in this limit then the observable depends on the cutoffs that in reality keep the background from becoming arbitrarily sharp and the coupling from becoming arbitrarily strong. Our prescription is: a) fix the background, b) compute the renormalized zero point energy, c) take the "boundary condition limit". This is to be contrasted with the standard approach: $a^{\prime}$ ) take the boundary condition limit, $b^{\prime}$ ) compute the zero point energy. The two approaches disagree for all surfaces (plates and spheres) and in all dimensions that we have studied. We believe that our prescription for the order of limits is the physical one, and that the result obtained by imposing the boundary condition $a b$ initio should be discarded when it disagrees with it.

Here we study the imposition of Dirichlet boundary conditions on a scalar field. It is straightforward to write down a QFT describing a renormalizable interaction between the fluctuating scalar field $\phi$ and a static, non-dynamical background field $\sigma(\mathbf{x})$,

$$
\mathcal{L}_{\text {int }}(\phi, \sigma)=-\frac{1}{2} \lambda \sigma(\mathbf{x}) \phi^{2}(\mathbf{x}, t)
$$

and to choose a limit involving the shape of $\sigma(\mathbf{x})$ and the coupling strength, $\lambda$, between $\phi$ and $\sigma$ that produces the desired boundary conditions on specified surfaces. The interaction strength is characterized by two parameters: the width $\Delta$ of the background and the strength $\lambda$. In the limit $\Delta \rightarrow 0$ the background becomes a surface $\delta$-function. We refer to this as the "sharp limit." Once the sharp limit has been taken, the limit $\lambda \rightarrow \infty$ enforces the Dirichlet boundary condition on all modes of the fluctuating field. We refer to this as the "strong" limit. The boundary condition emerges on the surface only in the limit that the background becomes both sharp and strong. Both $\Delta$ and $\lambda$ represent physical cutoffs characteristic of the material with which the fluctuating field interacts. We identify $\Delta$ with the physical thickness of the surface and $\lambda$ plays a role similar to the plasma frequency: modes with frequency much larger than the scale determined by $\lambda$ are not constrained at the boundary. Ideally, we seek a Casimir energy that reflects only the effects of the boundary conditions and not any other features of $\sigma(\mathbf{x})$. Therefore we do not specify any action for $\sigma$ except 


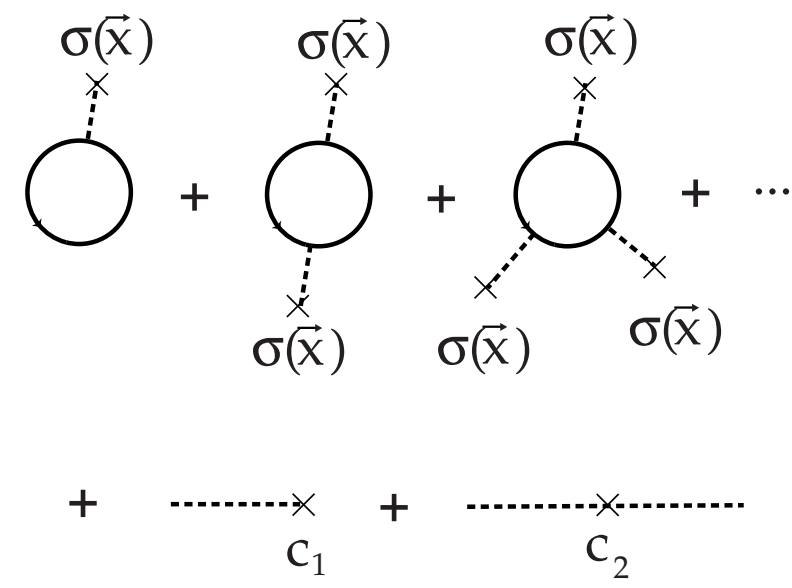

Figure 1: The Feynman graph series for the Casimir energy.

for the standard counterterms induced by the $\phi-\sigma$ interaction. The coefficients of the counterterms are fixed by renormalization conditions applied to perturbative Green's functions. Having been fixed in perturbation theory, the counterterms are fixed once and for all and must serve to remove the divergences that arise for any physically sensible $\sigma(\mathbf{x})$. Moreover, the renormalization conditions are independent of the particular choice of background $\sigma(\mathbf{x})$, so it makes sense to compare results for different choices of $\sigma(\mathbf{x})$, i.e. different geometries. In the simple theory we are considering, the only counterterms are $\mathcal{L}_{\mathrm{ct} 1}(\sigma)=c_{1} \sigma(\mathbf{x})$, which renormalizes the tadpole graph and, in three dimensions, $\mathcal{L}_{\mathrm{ct} 2}(\sigma)=c_{2} \sigma^{2}(\mathbf{x})$, which renormalizes the self-energy.

The vacuum polarization energy - the Casimir energy, $E[\sigma]$ - for the field $\phi$ in the background $\sigma$ is given by the sum of all one-loop Feynman diagrams plus the contributions of the counterterms, as shown in Fig. [1

The diagrams generate a representation for $E[\sigma]$ as a power series in $\lambda$. It is easy to show that

- for piecewise continuous $\sigma$ (characterized by a width $\Delta$ ), the contribution to the renormalized Casimir energy at each order in $\lambda$ is finite, and

- the sum over the terms in the power series in $\lambda$ gives a finite, renormalized Casimir energy for any fixed $\lambda$ and $\Delta$.

The first result is a general consequence of the renormalizability of the $\phi-\sigma$ field theory. The second result can be demonstrated explicitly by rewriting the Casimir energy as a 
sum/integral over scattering data (bound state energies and scattering phase shifts) in the fashion of Refs. 9, 10, 11, where it is manifestly finite.

The thickness $\Delta$ sets the scale for the Fourier components, $\tilde{\sigma}(\mathbf{p})=\int d^{D} x \sigma(\mathbf{x}) \exp (i \mathbf{p} \cdot \mathbf{x})$ in D space dimensions, of the background field, which are integrated in the one-loop diagrams of Fig. 1. As $\Delta \rightarrow 0$ the Fourier integrals in low-order diagrams diverge. This simple mechanism is responsible for the cutoff dependence of the Casimir energy. Because it occurs in low-order diagrams (order $\leq 3$ for three dimensions or less) we can examine the divergences with well-known and universally accepted methods of analysis [26]. This analysis forms the technical core of this paper.

The simplest diagram, the tadpole, is momentum independent. In our renormalization scheme it is canceled exactly by the counterterm $\mathcal{L}_{\text {ct } 1}$ leaving no contribution to the Casimir energy at order $\lambda$. The two-point function has a simple representation in terms of $\tilde{\sigma}(\mathbf{p})$. In two dimensions, we have

$$
E_{2 \mathrm{D}}^{(2)}[\sigma]=-\frac{\lambda^{2}}{16 \pi} \int \frac{d^{2} p}{(2 \pi)^{2}} \tilde{\sigma}(\mathbf{p}) \tilde{\sigma}(-\mathbf{p}) \frac{1}{|\mathbf{p}|} \tan ^{-1} \frac{|\mathbf{p}|}{2 m},
$$

and in three dimensions, after renormalization we have

$$
E_{3 \mathrm{D}}^{(2)}[\sigma]=\frac{\lambda^{2}}{64 \pi^{2}} \int \frac{d^{3} p}{(2 \pi)^{3}} \tilde{\sigma}(\mathbf{p}) \tilde{\sigma}(-\mathbf{p}) \int_{0}^{1} d x \ln \frac{m^{2}+x(1-x) \mathbf{p}^{2}}{m^{2}+x(1-x) \mu^{2}} .
$$

Note the dependence of $E_{3 \mathrm{D}}^{(2)}[\sigma]$ on the (spacelike) renormalization scale $\mu$. In the first part of Section 3 we show that these contributions to the Casimir energy diverge like $\ln \Delta$ and $(\ln \Delta) / \Delta$ in the sharp limit. There is no mechanism available to cancel these divergences. In two dimensions, if we use the counterterm $\mathcal{L}_{\text {ct } 2}(\sigma)$ with finite coefficient $c_{2}$ to make a finite renormalization of the two-point function, the divergence only gets worse, as discussed in Section 3.2. In three dimensions we have already renormalized the theory as the $\mu$ dependence of $E_{3 \mathrm{D}}^{(2)}[\sigma]$ attests and yet the divergence in the sharp limit persists. We show that these cutoff dependences affect the Casimir pressure.

The reader may still worry that some more subtle interpretation of renormalization will still succeed in removing the divergence, especially in $E_{3 \mathrm{D}}^{(2)}[\sigma]$ which had a primitive (loop) divergence prior to renormalization even for fixed $\Delta$. To address that concern we study the three-point function in three dimensions, for which there is no loop divergence. We show that this diagram also diverges (like $\ln \Delta$ ) in the sharp limit - the situation is analogous to the two-point function in two dimensions. There is no mechanism to remove this divergence at any stage in the formulation of the theory, and it contributes to the Casimir pressure as well. Thus the Casimir pressure on a sphere is unavoidably cutoff dependent both in two and three dimensions.

We have made many consistency checks on this calculation. In addition to the analytical analysis of the $\Delta \rightarrow 0$ limit given in Sections 3 and 4 , we have computed (in Section 5) 
the diagrams in the sharp limit with a $\delta$-function source but with a frequency cutoff on $\tilde{\sigma}(\mathbf{p})$. This is a simpler computation and it yields the same result. Finally we have used the "interface" formalism developed in Ref. [12] (in the Appendix) to generalize from one to two to three dimensions, again confirming the result of the direct analytical analysis.

The fact that the energy of a fluctuating field diverges when a boundary condition is imposed has been known for many years [13, 14. However divergences are common in quantum field theory and renormalization was invented to remove them. Special calculational methods have been developed to eliminate the extra divergences that arise in the presence of boundaries, which give finite, cutoff-independent Casimir energies [7]. These methods do not address the crucial question of whether the discarded divergences reflect physically significant cutoff dependence or are merely unphysical artifacts of the calculational method. Several studies of these divergences have been made. Symanzik studied the divergences of a scalar field theory in the presence of a surface interaction that imposes the boundary condition $\phi=0$ [15]. He showed that it is possible to introduce a set of surface counterterms sufficient to cancel all the divergences that arise in this theory. There are many independent counterterms and their forms are complex. However, the counterterms available in a renormalizable quantum field theory are few in number and simple in form, merely polynomials in the field and its derivatives. There is no freedom to introduce Symanzik's ad hoc surface counterterms if one views the boundary condition as arising from an underlying renormalizable quantum field theory (as it must). Therefore his methods cannot be used to explore the physical implications of the divergences generated by boundary conditions.

The work of Candelas [14 and Candelas and Deutsch [13 is similar to our both in spirit and conclusions. In Ref. [13] they studied the Casimir energy and energy density for scalar and electromagnetic fields in the presence of boundaries. Candelas later refined this work and extended it to boundaries between dielectrics. They point out that the vacuum polarization energy density generally diverges as one approaches a boundary and state that these divergences are not the ones that can be cancelled by standard renormalization methods. They note that the famous special cases where the divergences cancel - the conformal scalar field near a planar boundary [16] and the electromagnetic field near a plane or a sphere [17 are indeed exceptional. The cancellations fail and the Casimir energy density is divergent if the surface has (arbitrarily small) shape imperfections. Candelas and Deutsch speculate that divergences are generic and that a more realistic treatment of the boundary would confirm this. In Ref. [14] Candelas develops a model of a dielectric in order to examine the divergences in Casimir energies under more realistic conditions. He finds that divergences are generic. The divergences that arise in the dielectric case are worse than those found when conducting boundary conditions are applied $a b$ initio. Candelas warns that his results cast doubt on Casimir calculations which begin with idealized boundary conditions. However, in the years following Candelas's work this warning seems largely to have been ignored. The dielectric model used by Candelas does not correspond to a renormalizable continuum quan- 
tum field theory. ${ }^{1}$ Therefore one cannot state for certain which divergences can or cannot be cancelled by counterterms. Also, when he studies conductors or the Dirichlet problem, Candelas imposes boundary conditions ab initio, introducing the possibility of missing physically significant divergences. Our work can be regarded as a reformulation of Candelas's critique of Casimir calculations in the context of renormalizable quantum field theory. In this context there is no longer any reason to doubt the physical significance of the cutoff dependence we have found.

Finally our results disagree with the recent results of Ref. [18, which also considers loworder Feynman diagrams, but obtains a finite contribution to the stress in the boundary condition limit. The specific disagreements are discussed in the Conclusions after we have introduced the conceptual framework necessary for the discussion.

We note that some of these results have appeared in a brief introduction to our work 8] and in a conference presentation [19, where the one-dimensional case was discussed in complete detail. A summary of the two dimensional case was also given in [8]. The divergences of the Casimir energy in one dimension are less severe, but still prevent one from defining a Casimir energy in the Dirichlet boundary condition limit. Finally, we note that subtleties similar to the ones we have considered were addressed in the context of dispersive media in Ref. [20]

\section{General structure of the Casimir energy}

In this section we outline the calculation of the Casimir energy of a scalar field in a piecewise continuous scalar background field in two or three space dimensions $(D=2,3)$. We review the Feynman diagram expansion and renormalization issues and summarize the scattering data approach which sums all Feynman diagrams and gives an unambiguous renormalized vacuum polarization energy. We take a first look at how divergences can arise in the renormalized Casimir energy as the background becomes singular and identify the Feynman diagrams that play a central role.

We consider the theory of a real scalar field $\phi$ coupled to a time-independent background field $\sigma(\mathbf{x})$, described by the Lagrangian,

$$
\mathcal{L}=\frac{1}{2} \partial_{\mu} \phi \partial^{\mu} \phi-\frac{m^{2}}{2} \phi^{2}-\frac{\lambda}{2} \phi^{2} \sigma(\mathbf{x})+\mathcal{L}_{\mathrm{CT}}[\sigma] .
$$

$\mathcal{L}_{\mathrm{CT}}[\sigma]$ is the counterterm Lagrangian required by renormalization.

The Casimir energy is defined as the vacuum energy in the presence of $\sigma$ minus the vacuum energy in its absence. It can be written formally as the sum over the shifts in

\footnotetext{
${ }^{1}$ Candelas models the dielectric in terms of elementary electric and magnetic oscillators, which could in principle be embedded in a renormalizable theory. However the effective field theory obtained by integrating out the oscillators to obtain a dielectic is no longer renormalizable.
} 
the zero-point energies of all the modes of $\phi$ relative to the trivial background $\sigma=0$, $E_{\text {bare }}[\sigma]=\frac{1}{2} \sum_{n}\left(\omega_{n}[\sigma]-\omega_{n}^{(0)}\right)$. Equivalently, using the effective action formalism, $E_{\text {bare }}[\sigma]$ is given by the sum of all one-loop Feynman diagrams with at least one external $\sigma$ field, as shown in Fig. 1. The low-order diagrams generate divergences, which are canceled by the counterterms in $\mathcal{L}_{\mathrm{CT}}$. Combining its contribution to the energy, $E_{\mathrm{CT}}[\sigma]$, with $E_{\text {bare }}[\sigma]$ yields the renormalized Casimir energy $E[\sigma]$.

We have introduced $\sigma(\mathbf{x})$ as a non-dynamical, external field, solely to constrain $\phi$. In addition to the $\phi-\sigma$ coupling we include only the counterterms induced by the renormalization process. Of course one could always add non-trivial dynamics for $\sigma$ in the form $\mathcal{L}[\sigma]=$ $\frac{1}{2}(\partial \sigma)^{2}-V(\sigma)$. Such terms are undoubtedly present in a real material, where they correspond to the self-interactions of the material. However, since we want to know whether the vacuum energy associated with the fluctuations of $\phi$ can be isolated from the rest of the problem, we suppress any additional dynamics associated with $\sigma$.

Because we are calculating in a renormalizable quantum field theory, $E[\sigma]$ will be finite for any smooth $\sigma$ and finite $\lambda$. Renormalization is straightforward. First the theory is regulated. Next, superficially divergent Feynman diagrams are identified. Only the one-point function (the "tadpole") and the two-point function (the $\sigma$ self-energy) are superficially divergent for $D \leq 3$. The divergences are local and can therefore be canceled by counterterms of the form

$$
\mathcal{L}_{\mathrm{CT}}[\sigma]=c_{1}(\eta) \sigma+c_{2}(\eta) \sigma^{2}
$$

Here $\eta$ is a regulator, for example the deviation from the physical spacetime dimension in dimensional regularization. The constants $c_{1}$ and $c_{2}$ are fixed by renormalization conditions. We choose the "no tadpole" condition $\langle\sigma\rangle=0$ to fix $c_{1}$, and we require that the renormalized two-point function, $\bar{\Gamma}_{2}$, vanish at $p^{2}=-\mu^{2}$ to fix $c_{2}$. Of course any other choice of renormalization scheme can be related to ours by appropriate renormalization group transformations. We wish to stress that the renormalization process fixes the counterterms completely, independent of $\sigma$. So, for example, the counterterm $c_{1}$ is given by,

$$
c_{1}=\lambda \frac{m^{n-2}}{(4 \pi)^{\frac{n}{2}}} \Gamma\left(\frac{2-n}{2}\right)
$$

in $n$ spacetime dimensions. No ad hoc modifications of the counterterms can be introduced to cancel an unanticipated divergence.

Although the Casimir energy can be formally represented as the sum of all one-loop Feynman diagrams, only the first few can be computed directly. As the number of external lines increases so does the number of integrations over the external momenta carried by $\tilde{\sigma}(\mathbf{p})$, the Fourier transform of $\sigma(\mathbf{x})$. In practice it becomes tedious to compute beyond the three-point function directly. Furthermore the power series in $\lambda$ generated by the Feynman diagrams may not converge. We rely instead on methods that allows us to compute the 
Casimir energy of the background configuration exactly for any $\lambda$, while still performing all the necessary renormalization in the perturbative sector. The full Casimir energy can be computed exactly using phase shift methods, [9, 10, 11, by summing the derivative expansion [21, 22, or through analysis of Green's functions [11, 23]. All these methods show that once the counterterms corresponding to low-order diagrams have been included, the total Casimir energy $E[\sigma]$ for a smooth background is finite. Expanding this result in powers of $\lambda$, we recover the diagrammatic expansion and can isolate the effects of the counterterms.

Before analyzing the contributions of order $\lambda^{2}$ and $\lambda^{3}$ in detail, we give an overview of the possible divergences in $E[\sigma]$ when the background $\sigma$ becomes sharp and strong. We will consider a square barrier of width $\Delta$ and height $1 / \Delta$. Any smooth shape that approaches a surface delta function as $\Delta \rightarrow 0$ would do.

- The tadpole diagram is local, i.e. proportional to $\sigma(\mathbf{x})$, and therefore is canceled entirely by the counterterm $\mathcal{L}_{1}=c_{1} \sigma(\mathbf{x})$. The resulting rule for calculation is merely to drop the contribution of first order in $\lambda$ to the Casimir energy. No matter how singular the source, the contribution of order $\lambda$ to $E$ vanishes as a result of our choice of renormalization scheme.

- The contribution of order $\lambda^{2}$ comes entirely from the renormalized two-point function, which can be computed using standard methods. So the rule for calculation is to remove the terms of order $\lambda^{2}$ from the scattering data and replace them by $E^{(2)}[\sigma]$. The expressions for $E^{(2)}[\sigma]$ in two and three space dimensions have been given in eqs. (2) and eq. (3) respectively.

$E^{(2)}[\sigma]$ can diverge if the background $\sigma$ has a Fourier spectrum that vanishes too slowly at large $p$. This is precisely what happens when $\Delta \rightarrow 0$. Thus, examination of the renormalized two-point function alone is enough to establish that the renormalized Casimir energy diverges in the Dirichlet limit.

- The higher order (in $\lambda$ ) contributions to $E[\sigma]$ are guaranteed to be finite for smooth enough $\sigma$ and finite $\lambda$ because only the tadpole and self-energy diagrams have primitive divergences in the underlying field theory for $D \leq 3$. We find that $\sigma(\mathbf{x})$ which are piecewise continuous are smooth enough to give a finite renormalized Casimir energy. Thus the renormalized Casimir energy is finite for any fixed $\Delta$ and $\lambda$.

In two dimensions there are no divergences beyond the two-point function even in the sharp limit. However, in three dimensions as the background field becomes sharp, i.e. as $\Delta \rightarrow 0$, the three-point function diverges as well. In Section 4 we calculate the three-point function in the background of parallel plates of width $\Delta$ and also in the background of a spherical shell of with $\Delta$. We study the limit $\Delta \rightarrow 0$ and show that this contribution to $E$ diverges. 
In Section 5, we calculate the contribution to the vacuum polarization energy at $n^{\text {th }}$ order in $\lambda$ for a surface $\delta$-function in three dimensions for both plane and spherical geometries. We find a result that is finite for $n>3$, but diverges logarithmically for $n=3$, a divergence that cannot have anything to do with choice of renormalization scheme or other subtleties.

To summarize: Potential divergences in the Casimir energy in the Dirichlet limit are isolated in the two-point function in two dimensions and in the two and three-point functions in three dimensions. These contributions are analyzed in depth in the next two sections for single plate, parallel plate and spherical geometries in two and three dimensions.

\section{The Two-Point Function}

In this section and the next we analyze the low-order Feynman diagrams that are potentially divergent in the sharp limit, $\Delta \rightarrow 0$. We treat the case of a square barrier, although any one-parameter family of functions which approaches a $\delta$-function as $\Delta \rightarrow 0$ would do. In this section we study the renormalized two-point function as defined in eqs. (21) and (3). While this analysis alone is sufficient to establish a divergence in the total energy, the fact that in three space dimensions it requires renormalization may make some readers uneasy. The three-point function, while more difficult to compute, has no such complication and we will study its divergences in the next section.

We study three geometries: a single plate

$$
\sigma_{\mid}(z)=\frac{1}{\Delta}(\theta(z+\Delta / 2)-\theta(z-\Delta / 2))
$$

parallel plates separated by a distance $2 L$ (both configurations in $\mathrm{D}(\leq 3)$ space dimensions),

$$
\sigma_{\|}(z)=\frac{1}{\Delta}(\theta(|z|-L+\Delta / 2)-\theta(|z|-L-\Delta / 2)),
$$

and a sphere of radius $R$ in $\mathrm{D}=3$ space dimensions,

$$
\sigma_{\circ}(r)=\frac{3}{4 \pi\left((R+\Delta)^{3}-R^{3}\right)}(\theta(r-R)-\theta(r-R-\Delta)) .
$$

We have chosen to normalize $\sigma$ analogously for plates and spheres. For plates we have normalized $\sigma$ so its integral across each plate is proportional to the surface area and independent of the width $\Delta$ and the number of dimensions, i.e. we require $\int d z \sigma(z)=N$, where $N=1$ or 2 , is the number of plates present. For $\sigma_{\mid}$and $\sigma_{\|}$we study $\mathcal{E}$, the Casimir energy 
per unit area in three dimensions or per unit length in two dimensions. In the case of a spherical shell of radius $R$ we have normalized $\sigma$ so that

$$
\int d^{3} x \sigma_{\circ}(\mathbf{x})=1
$$

independent of the width $\Delta$. We are aware of the fact that different normalization choices imply different dimensions for $\lambda$. These can be easily introduced, for example, by introducing appropriate powers of $m$, the mass of the fluctuating particle.

The choice of normalization has little significance for single or parallel plates. However it does have physical consequences when we measure the stress on a circular or spherical shell. To measure the stress, it is necessary to compare the energies of shells of radius $R$ and $R+\delta R$. To make this comparison, one must decide how the matter behaves as $R$ changes. Unfortunately, one of the shortcomings of the traditional boundary condition approach is that it tells us nothing about the physical properties of the material. So we must consider a range of possible behaviors. With the normalization chosen above, $\int d r \sigma_{\circ}(r) \sim 1 / R^{2}$ in three dimensions. This normalization corresponds to keeping the volume of $\sigma$ fixed as the shell is expanded, as if it were a physical substance. Another possibility would be to hold $\int d r \sigma_{\circ}(r)$ fixed as $R$ varies. We consider both possibilities when discussing the stress on a shell. We will see that it is not possible to choose any normalization of $\sigma$ that would eliminate divergences in the renormalized Casimir stress on a sphere in three dimensions.

It is convenient to work in momentum space when computing n-point functions. The background fields in momentum space are given by the Fourier transforms of eqs. (77), (8), (9) in the case of the single plate, parallel plates and sphere respectively:

$$
\begin{aligned}
& \tilde{\sigma}_{\mid}(\mathbf{q})=\Pi_{i=1}^{D-1}\left[(2 \pi) \delta\left(q^{i}\right)\right] \frac{2}{q_{z} \Delta} \sin \left(\frac{q_{z} \Delta}{2}\right), \\
& \tilde{\sigma}_{\|}(\mathbf{q})=\Pi_{i=1}^{D-1}\left[(2 \pi) \delta\left(q^{i}\right)\right] \frac{4}{q_{z} \Delta} \cos \left(q_{z} L\right) \sin \left(\frac{q_{z} \Delta}{2}\right), \\
& \tilde{\sigma}_{\circ}(\mathbf{q})=\frac{3}{q^{3}} \frac{q R[\cos (q R)-(1+\Delta / R) \cos (q(R+\Delta))]-\sin (q R)+\sin (q(R+\Delta))}{(R+\Delta)^{3}-R^{3}},
\end{aligned}
$$

where $q=|\mathbf{q}|$.

Most of our interest lies in three dimensions where there has been some controversy about the nature of the divergences in this problem [18. At the end of this section we present analogous results in two dimensions for comparison. The two-point function contribution to the Casimir energy can be computed analytically for a massless scalar field. Therefore we begin with the massless case. 


\subsection{Massless Case}

In two dimensions the two-point function contribution to the Casimir energy is infrared divergent in the massless case. So in this subsection we confine ourselves to three dimensions. In three dimensions the renormalized two-point function was given in eq. (33), and simplifies in the massless case to

$$
E_{3 \mathrm{D}}^{(2)}[\sigma]=\frac{\lambda^{2}}{64 \pi^{2}} \int \frac{d^{3} p}{(2 \pi)^{3}} \tilde{\sigma}(\mathbf{p}) \tilde{\sigma}(-\mathbf{p}) \ln \frac{\mathbf{p}^{2}}{\mu^{2}},
$$

For the single plate, eq. (77), we obtain

$$
\begin{aligned}
\mathcal{E}_{3 \mathrm{D}}^{(2)}\left(\sigma_{\mid}\right) & =\frac{\lambda^{2}}{16 \pi^{2} \Delta^{2}} \int_{-\infty}^{\infty} \frac{d p}{2 \pi} \frac{1}{p^{2}} \sin ^{2}(p \Delta / 2) \ln \left(p^{2} / \mu^{2}\right) \\
& =-\frac{\lambda^{2}}{32 \pi^{2} \Delta}(\ln (\mu \Delta)+\gamma-1),
\end{aligned}
$$

for the energy per unit area, where $\gamma=0.577 \ldots$ is Euler's constant. From this we conclude that the Casimir energy per unit area of a single plate diverges as $\frac{1}{\Delta} \ln \Delta$ in the sharp limit, $\Delta \rightarrow 0$. This is the first example of a general result: if we normalize the background field $\sigma$ to the surface area of the body under consideration, then the form of the leading and the first subleading divergence is always

$$
E_{3 \mathrm{D}}^{(2)}=-\frac{\lambda^{2}}{32 \pi^{2}} \frac{A}{\Delta}(\ln (\mu \Delta)+\gamma-1)
$$

where $A$ is the area. In the cases of single and parallel plates there are no further divergences

in the sharp limit, but in the case of a sphere there is a further sub-subleading divergence that is not universal.

Next consider the case of parallel plates, eq. (8),

$$
\mathcal{E}_{3 \mathrm{D}}^{(2)}\left(\sigma_{\|}\right)=\frac{\lambda^{2}}{8 \pi^{2} \Delta^{2}} \int_{-\infty}^{\infty} \frac{d p}{2 \pi} \frac{1}{p^{2}}(1+\cos (2 p L)) \sin ^{2}(p \Delta / 2) \ln \left(p^{2} / \mu^{2}\right)
$$

This integral can be carried out by deforming the integration contour in the complex plane,

$$
\begin{gathered}
\mathcal{E}_{3 \mathrm{D}}^{(2)}\left(\sigma_{\|}\right)=-\frac{\lambda^{2}}{16 \pi^{2} \Delta}\left\{\ln (\Delta \mu)+\gamma-1+\left(\frac{1}{2}+\frac{L}{\Delta}\right) \ln (1+\Delta / 2 L)\right. \\
\left.-\left(\frac{1}{2}-\frac{L}{\Delta}\right) \ln (1-\Delta / 2 L)\right\}
\end{gathered}
$$

It is easy to check that this result has the proper behavior as the plates separate to infinity or coalesce. As $L \rightarrow \infty$, the energy per unit area approaches twice the result for a single plate of 
unit strength (see eq. (15)). And as the plates coalesce at $L=\Delta / 2, \mathcal{E}_{3 \mathrm{D}}^{(2)}\left(\sigma_{\|}\right)$becomes equal to the energy per unit area of a single plate of strength two with width $2 \Delta$, as expected. As expected, the divergence in $\mathcal{E}_{3 \mathrm{D}}^{(2)}\left(\sigma_{\|}\right)$is twice that in $\mathcal{E}_{3 \mathrm{D}}^{(2)}\left(\sigma_{\mid}\right)$.

Although the Casimir energy for two parallel plates diverges as $\Delta \rightarrow 0$, the divergent terms are independent of $L$, explicitly,

$$
\mathcal{E}_{3 \mathrm{D}}^{(2)}\left(\sigma_{\|}\right)=-\frac{\lambda^{2}}{16 \pi^{2} \Delta}\left\{\ln (\Delta \mu)+\gamma-1+\frac{\Delta}{4 L}+\mathcal{O}\left(\frac{\Delta^{2}}{L^{2}}\right)\right\}
$$

and therefore the two-point contribution to the Casimir force remains finite in the sharp limit. Also, only the $L$-independent terms depend on the renormalization scale $\mu$. That is, this force does not have renormalization ambiguities.

Next, we turn to the spherical shell. Using eq. (91), we obtain,

$$
\begin{aligned}
E_{3 \mathrm{D}}^{(2)}\left(\sigma_{\circ}\right)= & -\frac{\lambda^{2}}{256 \pi^{3} R \Delta^{2}\left(1+\epsilon+\frac{1}{2} \epsilon^{2}\right)^{2}}\left\{\frac{2}{3} \ln \frac{4(1+\epsilon)}{(2+\epsilon)^{2}}-2 \epsilon \ln \frac{2+2 \epsilon}{2+\epsilon}\right. \\
& +2 \epsilon(\ln (\mu \Delta)+\gamma-1)+\epsilon^{2}\left(-\frac{4}{3}+2 \ln \frac{2+2 \epsilon}{2+\epsilon}+2(\ln (\mu \Delta)+\gamma-1)\right) \\
& \left.+\epsilon^{3}\left(-\frac{8}{9}+\frac{2}{3} \ln \frac{2+2 \epsilon}{2+\epsilon}+\frac{2}{3}(\ln (\mu \Delta)+\gamma-1)\right)\right\} .
\end{aligned}
$$

where $\epsilon=\Delta / R$. The terms that diverge in the limit $\Delta \rightarrow 0$ can be singled out

$$
E_{3 \mathrm{D}}^{(2)}\left(\sigma_{\circ}\right)=-\frac{\lambda^{2}}{128 \pi^{3} R^{2}}\left(\frac{1}{\Delta}(\ln (\mu \Delta)+\gamma-1)-\frac{\ln (\mu \Delta)}{R}\right)+\text { terms finite for } \Delta \rightarrow 0 .(21
$$

If we correct for the different normalizations, we find that the leading and the first subleading divergent contribution is the same (per unit area) as for the plate(s). However its physical consequences are quite different. In the case of an isolated sphere the stress,

$$
\mathcal{P}_{3 \mathrm{D}}^{(2)}\left(\sigma_{\circ}\right)=-\frac{\partial E_{3 \mathrm{D}}^{(2)}\left(\sigma_{\circ}\right)}{\partial R}
$$

is the quantity of interest. Apparently the stress diverges as $\frac{1}{R^{3} \Delta} \ln \Delta$ as $\Delta \rightarrow 0$. Had we normalized $\sigma_{\circ}$ in the same way as $\sigma_{\mid}$and $\sigma_{\|}$, so that $\int d r \sigma_{\circ}(r) \sim$ constant, the $R$ dependence of the prefactor in eq. (21) would have changed from $\propto 1 / R^{2}$ to $\propto R^{2}$. The stress changes sign, however it would still diverge as $\Delta \rightarrow 0$. This highlights the fact that the stress depends in detail on the surface dynamics.

The reader might be tempted to eliminate the $R$ dependence from the divergences in eq. (21) by choosing a different normalization for the background $\sigma$ and redefining $R \rightarrow R+\Delta / 2$. Such a transformation is possible, and would render the Casimir pressure from the two-point function finite. However, as we shall see below, the three-point function also contributes a divergent Casimir pressure, and no transformations can eliminate all of the divergences. 


\subsection{Case of Non-zero Mass}

\section{Three Dimensions}

It has been claimed that the divergences in the sharp limit are an artifact of the specialization to massless bosons [18. It is certainly true that low-order Feynman diagrams have infrared divergences in low dimension. However these are not the divergences that plague the Casimir energy. To show that the divergences of the previous section persist when $m \neq 0$ we present the case of a single plate. The other geometries of interest behave analogously.

For a scalar boson of mass $m$ in three dimensions we have to evaluate

$$
\begin{aligned}
& \mathcal{E}_{3 \mathrm{D}}^{(2)}\left(\sigma_{\mid}\right)= \frac{\lambda^{2}}{16 \pi^{2} \Delta^{2}} \int_{-\infty}^{\infty} \frac{d p}{2 \pi} \frac{1}{p^{2}} \sin ^{2}(p \Delta / 2) \int_{0}^{1} d x \ln \left(\frac{m^{2}+x(1-x) p^{2}}{m^{2}+x(1-x) \mu^{2}}\right) \\
&=-\frac{\lambda^{2}}{32 \pi^{2} \Delta}\left\{\ln (\mu \Delta)-1-\frac{1}{m \Delta} \int_{2}^{\infty} d \xi \frac{\sqrt{\xi^{2}-4}}{\xi^{3}}\left(1-e^{-\xi m \Delta}\right)-\ln (m \Delta)\right. \\
&\left.+\frac{1}{2} \int_{0}^{1} d x \ln \left(1+\frac{m^{2} / \mu^{2}}{x(1-x)}\right)\right\} .
\end{aligned}
$$

Note that the massless $(m \rightarrow 0)$ and $\operatorname{sharp}(\Delta \rightarrow 0)$ limits coincide in the $\xi$ integral term and that

$$
\lim _{\eta \rightarrow 0}\left(\frac{1}{\eta} \int_{2}^{\infty} d \xi \frac{\sqrt{\xi^{2}-4}}{\xi^{3}}\left(1-e^{-\xi \eta}\right)+\ln \eta\right)=-\gamma .
$$

In addition, the last term of eq. (23), the Feynman parameter integral, vanishes in the massless limit. So the leading $\Delta \rightarrow 0$ divergence of $\mathcal{E}_{3 \mathrm{D}}^{(2)}\left(\sigma_{\mid}\right)$is the same whether or not $\phi$ has a mass. This is true for all three geometries we have studied. Note that the subleading divergence proportional to $\frac{1}{\Delta}$ is altered in the case of a massive scalar boson.

When the mass of $\phi$ is non-zero it is possible to study the two dimensional case. This problem has been studied with a Gaußian background in [11. For comparison with the threedimensional case, we compute the Casimir energy of a single plate (line) in two dimensions with the background of eq. (8).

\section{Two Dimensions}

In two spatial dimensions the vacuum polarization diagram is finite and requires no renormalization. However, the contribution to the energy becomes infinite in the sharp limit, as we will now show. The integration in eq. (22) cannot be performed analytically for arbitrary $\Delta$. However the leading behavior as $\Delta \rightarrow 0$ can be easily obtained. The energy per unit length is given by

$$
\mathcal{E}_{2 \mathrm{D}}^{(2)}\left(\sigma_{\mid}\right)=\frac{\lambda^{2}}{32 \pi} \ln (\Delta m)+\text { terms finite for } \Delta \rightarrow 0
$$


This calculation demonstrates that the contribution to the energy from the second-order Feynman diagram in two spatial dimensions is divergent in the limit $\Delta \rightarrow 0$, and this divergence cannot be renormalized away. If we try to renormalize the vacuum polarization graph (by a finite counterterm proportional to $\sigma^{2}$ ), the divergence only gets worse. The two-point function

$$
\Pi(|\mathbf{p}|)=\frac{1}{4 \pi|\mathbf{p}|} \tan ^{-1} \frac{|\mathbf{p}|}{2 m}
$$

is renormalized to

$$
\Pi_{\text {ren }}(|\mathbf{p}|)=\Pi(|\mathbf{p}|)-\Pi(\mu)
$$

where $\mu$ is a renormalization scale and the leading divergence now is proportional to $1 / \Delta$. A numerical analysis of the vacuum polarization energy for a Gaußian background peaked on a circle of radius $R$ can be found in Ref. [11. There the energy density is studied, and the log-divergence that develops as $\Delta \rightarrow 0$ can be seen to be concentrated on the emerging surface at $R$.

\subsection{Comments}

At this point already some important conclusions may be drawn: first, the renormalized second order Feynman diagram diverges in the sharp limit for all the simple geometries we have considered. Second, since the Feynman diagram expansion is an expansion in powers of $\lambda$, the contribution from the second order diagram cannot be canceled by the other diagrams in the series. They are of different powers in $\lambda$. Third, the diagram has been renormalized, and the counterterm has been fixed. Thus, the divergence in the sharp limit is physical and continuum quantum field theory does not provide a way to get rid of it. If one is only interested in forces that do not require the body to be deformed, one need not be troubled by cutoff dependence in the energy. However, if one has to vary the surface size or geometry as in the case of a stress, the sharp limit gives a divergent result. It is already clear at this point that throwing away divergent terms on an ad hoc basis will eliminate parts of the physical measurable stress; therefore, in cases where one has to deform the body to obtain the stress the sharp limit cannot be taken.

\section{The Three-Point Function}

To further clarify the nature of the divergences in the Dirichlet Casimir energy, we consider the three-point function in a scalar theory in three dimensions. No divergences originate

from the loop integrations in the Feynman diagrams at this order (and beyond). As a result, 
we do not need to discuss the subtle issue of regularization and renormalization and no divergence in the three-point function can be renormalized away. It must instead reflect a physical cutoff dependence generated by the interaction of the fluctuating field with the background matter.

The three-point function for a fluctuating boson field reduces to

$$
E_{3 \mathrm{D}}^{(3)}[\sigma]=\frac{i \lambda^{3}}{3 T} \int \frac{d^{4} q_{1}}{(2 \pi)^{4}} \frac{d^{4} q_{2}}{(2 \pi)^{4}} \frac{d^{4} q_{3}}{(2 \pi)^{4}} \frac{\tilde{\sigma}_{4}\left(q_{1}-q_{2}\right)}{q_{2}^{2}-m^{2}+i \eta} \frac{\tilde{\sigma}_{4}\left(q_{2}-q_{3}\right)}{q_{3}^{2}-m^{2}+i \eta} \frac{\tilde{\sigma}_{4}\left(q_{3}-q_{1}\right)}{q_{1}^{2}-m^{2}+i \eta} .
$$

Here $\tilde{\sigma}_{4}(q)=\int d^{4} x \sigma(x) \mathrm{e}^{i q \cdot x}=2 \pi \delta\left(q^{0}\right) \tilde{\sigma}(\mathbf{q})$ is the Fourier transform of the time independent background field $\sigma(x)$ in four dimensions. The factor of $\frac{1}{T}$, where $T$ is a large time interval, converts the effective action for arbitrary $\sigma$ to the Casimir energy for time independent $\sigma$.

We consider two cases for $\sigma(\mathbf{x})$ : First we assume a spherically symmetric barrier of radius $R$ and width $\Delta$, given by eq. (9), and second, we consider parallel plates of width $\Delta$ separated by $2 L$, given by eq. (8).

As already noted, the integral over the loop momentum in eq. (28) converges. Thus $E_{3 \mathrm{D}}^{(3)}[\sigma]$ can be evaluated without reference to regularization. It can be cast into the form

$$
E_{3 \mathrm{D}}^{(3)}[\sigma] \propto \int_{0}^{\infty} d P f(P)
$$

where it is understood that $E_{3 \mathrm{D}}^{(3)}[\sigma]$ is to be replaced by the energy per unit area, $\mathcal{E}_{3 \mathrm{D}}^{(3)}[\sigma]$, in the case of parallel plates. In eq. (29) and below we omit constants of proportionality that are independent of the parameters $(R, L, \Delta)$ characterizing the background field, which are irrelevant for the present discussion. For simplicity of notation we also suppress the dependence of $E$ and $f$ on $R, L, \Delta, \lambda$, etc.

For the spherically symmetric background we find the integrand

$$
\begin{aligned}
& f_{\circ}(P)=P^{4} \int_{0}^{\frac{\pi}{2}} d \theta \cos \theta \sin ^{2} \theta \sigma_{\circ}(P \cos \theta) \sigma_{\circ}(P \sin \theta) \\
& \times \int_{-1}^{1} d z \sigma_{\circ}(P \sqrt{1+z \sin 2 \theta}) g(P \cos \theta, P \sin \theta, z)
\end{aligned}
$$

where

$$
\begin{aligned}
g(p, q, z)=\int_{0}^{1} \frac{d x}{\sqrt{4 m^{2}+4 x(1-x) q^{2}+(p+2 x q z)^{2}}} \\
\times\left\{\tanh ^{-1} \frac{2 x p-p+2 x q z}{\sqrt{4 m^{2}+4 x(1-x) q^{2}+(p+2 x q z)^{2}}}\right. \\
\left.\quad-\tanh ^{-1} \frac{p+2 x q z}{\sqrt{4 m^{2}+4 x(1-x) q^{2}+(p+2 x q z)^{2}}}\right\} .
\end{aligned}
$$




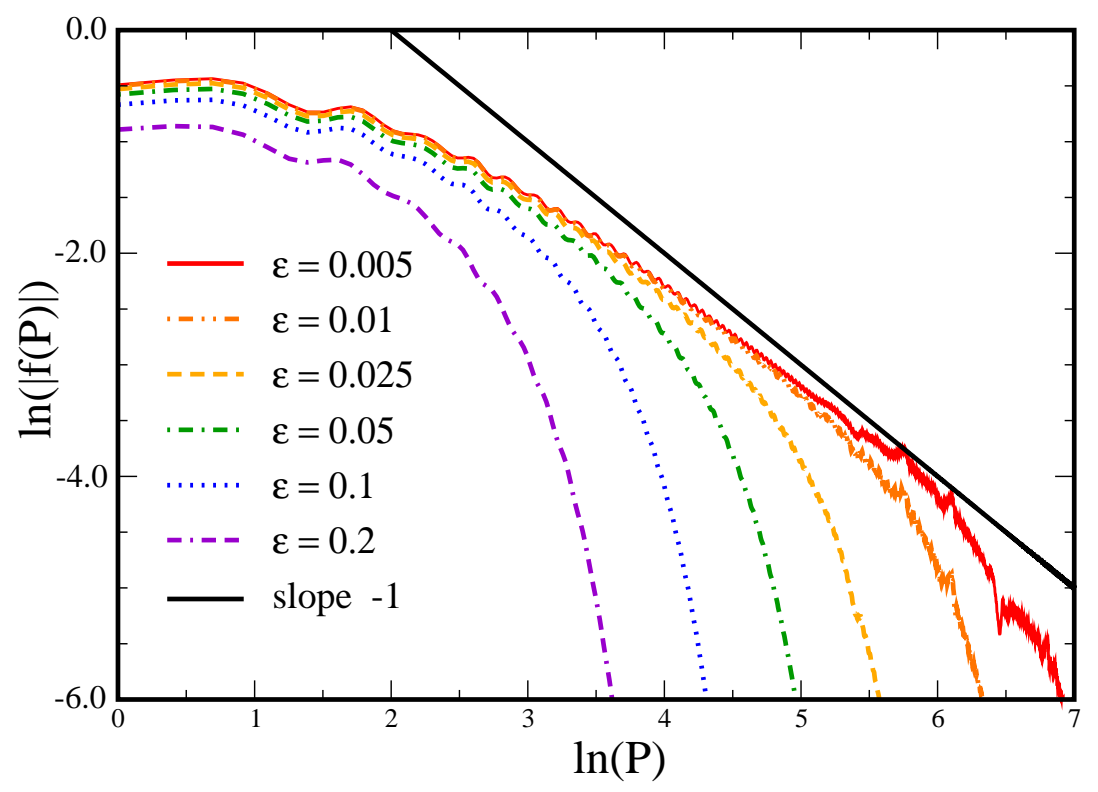

Figure 2: The logarithm of the integrand, eq. (30) of the three-point function for the spherical background, eq. (13) for various values of the thickness, $\epsilon=\Delta / R$, of the shell. Energies are measured in $m$, the mass of the particle in the loop. For orientation we have also included a limiting function, $f(P) \sim 1 / P$, for which $E^{(3)}$ diverges. Here the case $R=0.8 / m$ is shown.

The integrals in eqs. (29) and (30) cannot be evaluated analytically so we study them numerically.

In Fig. 2 we display a typical example for the integrand of the spherical problem, eq (30). For a given non-zero width, $\Delta$, there is always an intermediate region in which $f_{\circ}(P) \propto 1 / P$. Beyond that region the integrand drops off faster than $1 / P$ so the integral converges for finite width $\Delta$. As the width decreases, the region in which $f_{\circ}(P) \propto 1 / P$ increases and continues to larger values in $P$. As $\Delta \rightarrow 0$, this behavior persists for arbitrarily large $P$ and therefore the integral eq (29) diverges in that limit. The upper boundary of the region that exhibits the $1 / P$ behavior increases approximately like $1 / \Delta$. We can therefore estimate the divergence as a function of the width,

$$
\int_{0}^{\infty} d P f_{\circ}(P) \approx \int_{0}^{P_{0}} d P f_{\circ}(P)+\int_{P_{0}}^{1 / \Delta} d P \frac{C}{P}=C \ln \Delta+\ldots
$$

where $C$ is a constant and $P_{0}$ denotes the momentum at which the $1 / P$ behavior sets in. This logarithmic divergence is expected from the discussion above: in essence, $1 / \Delta$ serves as a large momentum cutoff on $f_{\circ}(P)$.

This divergence is studied in Fig. 圆 where we show $E_{3 \mathrm{D}}^{(3)}\left(\sigma_{\circ}\right)$ as function of the width parameter for various values of the position of the spherical barrier. Over the considered 


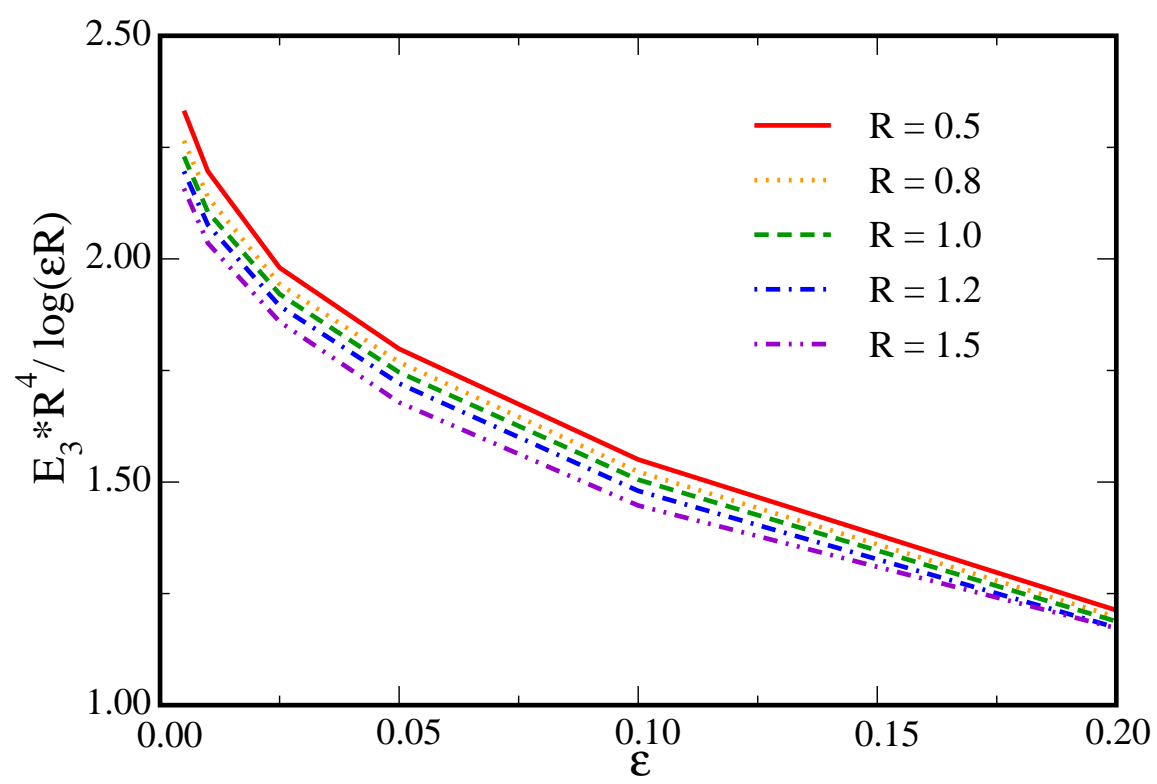

Figure 3: The contribution of the three-point function to the energy as a function of $\epsilon=\Delta / R$. Energies are measured in units of the mass $m$ of the particle in the loop. In those units the coupling constant $\lambda$ is chosen to be 1 and a scale is chosen to stress the $R$-dependence as well as the logarithmic dependence on $\Delta$.

range, $\Delta$ varies by about two orders of magnitude. On that scale the curves shown Fig. 3 are approximately constant and thus verify the logarithmic divergence estimated in eq. (31). So the divergence is not as severe as for the renormalized two-point function, but it is still present. As illustrated in the next section, where we discuss the $\delta$-function background (ie. $\Delta \rightarrow 0$ ), this is the expected behavior. When we restore the dimensional factors we see that $E_{3 \mathrm{D}}^{(3)}\left(\sigma_{\circ}\right) \propto \frac{1}{R^{4}} \ln \Delta$, so the constant of proportionality is $R$ dependent. Therefore the stress $\left(-\partial E_{3 \mathrm{D}}^{(3)}\left(\sigma_{\circ}\right) / \partial R\right)$ also diverges logarithmically as $\Delta \rightarrow 0$. As discussed in the previous section, the exact form of the $R$ dependence depends on the normalization we choose for $\sigma_{\circ}$. If we switch to the normalization used for plates where $\int d r \sigma(r)=1$, then $E_{3 \mathrm{D}}^{(3)}\left(\sigma_{\circ}\right) \propto R^{2} \ln \Delta$, so the divergent contribution to the stress, and even its sign(!), depends on the physical interpretation we place on $\sigma$, which is by no means clear. Note that the $R$ dependence of this divergence differs from that of the two-point function, so for every choice of normalization of $\sigma$ the sum of the two terms diverges.

The case in which the barriers approximate two parallel plates is simpler because one more integral can be evaluated analytically. The corresponding integrand in eq. (29) then 
reads,

$$
f_{\|}(P)=P \int_{0}^{2 \pi} d \theta \Sigma(P \cos \theta, P \sin \theta) g(P \cos \theta, P \sin \theta)
$$

where

$$
\begin{aligned}
\Sigma(p, q) & =\sigma_{\|}(p) \sigma_{\|}(q) \sigma_{\|}(p+q) \\
\sigma_{\|}(q) & =\frac{2}{q \Delta} \cos (q L) \sin \left(\frac{q \Delta}{2}\right)
\end{aligned}
$$

and

$$
\begin{aligned}
g(p, q)=\frac{1}{p} \int_{0}^{1} & \frac{d x}{\sqrt{4 m^{2}+4 x(1-x) q^{2}+(p+2 x q)^{2}}} \\
& \times\left\{\tanh ^{-1} \frac{2 x p-p+2 x q}{\sqrt{4 m^{2}+4 x(1-x) q^{2}+(p+2 x q)^{2}}}\right. \\
& \left.-\tanh ^{-1} \frac{p+2 x q}{\sqrt{4 m^{2}+4 x(1-x) q^{2}+(p+2 x q)^{2}}}\right\} .
\end{aligned}
$$

In Table 1 we present numerical results for the energy per unit area, $\mathcal{E}_{3 \mathrm{D}}^{(3)}\left(\sigma_{\|}\right)$, which originates from the three-point function. Like the sphere, $\mathcal{E}_{3 \mathrm{D}}^{(3)}\left(\sigma_{\|}\right)$diverges like $\ln \Delta$ as $\Delta \rightarrow 0$. However, this time the divergence is independent of $L$. As we found for the two-point function, the force between parallel plates due to the three-point function is finite and unambiguous as $\Delta \rightarrow 0$.

To summarize, we have found that the contribution of the three-point function to the Casimir energy of a sphere or the Casimir energy per unit area of parallel plates diverges logarithmically in the sharp limit in three dimensions. We expect that this divergence, like that of the two-point function, is the same for all shapes. This is because the width parameter essentially plays the role of a cutoff on the Fourier spectrum of the background, independent of its geometry. There is no counterterm in the continuum field theory to cancel this divergence. The stress on a sphere suffers the same divergence. However the force between parallel plates does not. Instead it reaches a finite limit as $\Delta \rightarrow 0$. In the case of the sphere, the $R$ dependence of $\sigma_{\circ}(r)$ could be chosen to render the divergence independent of $R$. However no choice can remove the divergences in both the two and three-point functions. We conclude that the Dirichlet-Casimir stress on a sphere in three dimensions depends in an unavoidable way on the cutoffs that characterize the material. It is not possible to compute even the cutoff dependent term in the stress on a sphere, to say nothing of the finite parts, because it depends dramatically on what is assumed about the $R$ dependence of $\sigma_{\circ}(r)$. 


\begin{tabular}{l|ccccc}
$\Delta$ & $L=0.5$ & $L=0.8$ & $L=1.0$ & $L=1.2$ & $L=1.5$ \\
\hline 0.010 & -163.04 & -160.24 & -160.88 & -161.04 & -161.28 \\
0.025 & -122.16 & -119.68 & -119.68 & -119.76 & -120.00 \\
0.050 & -95.36 & -92.64 & -92.16 & -92.08 & -92.24 \\
0.100 & -70.56 & -67.84 & -67.36 & -67.28 & -67.20 \\
0.200 & -48.08 & -44.72 & -45.12 & -45.04 & -44.96
\end{tabular}

Table 1: The contribution of the three-point function to the energy per unit area, $\mathcal{E}^{(3)}\left(\sigma_{\|}\right)$, as a function of the width, $\Delta$, and the distance of the parallel plates, L. Units are set by the mass, $m$, of the particle in the loop. Constants of proportionality, that do not vary with either $\Delta$ or $L$ are omitted. The variation of $\mathcal{E}^{(3)}\left(\sigma_{\|}\right)$across the columns of the Table is consistent within the accuracy of the calculation, with the divergent part of $\mathcal{E}^{(3)}\left(\sigma_{\|}\right)$being independent of $L$.

\section{Vacuum Polarization Energy in a Sharp Background}

In this section we take a different approach to the calculation of the Casimir energy in a sharp background in three space dimensions. Instead of introducing a finite thickness, $\Delta$, as a cutoff, we start with a $\delta$-function background, $\sigma^{*}$, from the start and introduce a cutoff, $\Lambda$, on its Fourier spectrum. This enables us to compute all $n$-point functions for the parallel plate and spherical geometries and to explore the divergences in a simpler mathematical context.

We first consider the case of parallel plates. To begin we impose the constraint that $\sigma^{*}$ depends only on $x_{3}$ and is symmetric under reflection in this coordinate,

$$
\sigma_{\|}^{*}(\mathbf{x})=\sigma_{\|}^{*}\left(x_{3}\right), \quad \sigma_{\|}^{*}\left(x_{3}\right)=\sigma_{\|}^{*}\left(-x_{3}\right) .
$$

The appropriate form of the free Green's function for this problem reads

$$
G_{0}\left(x, x^{\prime}\right)=\int \frac{d^{3} q}{(2 \pi)^{3}} \mathrm{e}^{i \tilde{q} \cdot\left(\tilde{x}-\tilde{x}^{\prime}\right)} \int_{-\infty}^{\infty} \frac{d p}{2 \pi} \sum_{\ell=0,1} \frac{\cos \left(x_{3} p+\frac{\ell \pi}{2}\right) \cos \left(x_{3}^{\prime} p+\frac{\ell \pi}{2}\right)}{\tilde{q}^{2}-p^{2}-m^{2}+i \eta} .
$$

Here $\tilde{v}_{\mu}=\left(v_{0}, v_{1}, v_{2}\right)$ comprises the time component as well as those spatial components of a four vector, $v_{\mu}$, orthogonal to $x_{3}$. The quantum number $\ell=0,1$ is associated with parity. The $n$-point function, $\Gamma^{(n)}\left(\sigma_{\|}^{*}\right)$, is given by

$$
\Gamma^{(n)}\left(\sigma_{\|}^{*}\right)=\frac{-i \lambda^{n}}{2 n} \int d^{4} x_{1} \int d^{4} x_{2} \ldots \int d^{4} x_{n} G\left(x_{1}, x_{2}\right) \sigma_{\|}^{*}\left(\mathbf{x}_{2}\right) G\left(x_{2}, x_{3}\right) \sigma_{\|}^{*}\left(\mathbf{x}_{3}\right) \ldots G\left(x_{n}, x_{1}\right) \sigma_{\|}^{*}\left(\mathbf{x}_{1}\right) .
$$


We have already established that $\Gamma^{(n)}\left(\sigma_{\|}^{*}\right)$ diverges for $n=1,2$, and 3 . They should be regarded as regulated by a cutoff which will be introduced explicitly below. For a background of the structure given in eq (33) the integrals $\int d^{3} \tilde{x}_{i}$ are straightforward $\left(\tilde{x}=\left(x^{0}, x^{1}, x^{2}\right)\right)$. They yield overall (infinite) factors of the "time interval" and "area" of the plates. The energy per unit area is obtained by dividing out these factors. The order $n$ contribution is (after Wick rotation)

$$
\begin{aligned}
\mathcal{E}_{3 \mathrm{D}}^{(n)}\left(\sigma_{\|}^{*}\right)=- & \frac{(-\lambda)^{n}}{2 n} \int \frac{d^{3} \tilde{q}}{(2 \pi)^{3}} \int_{-\infty}^{\infty} \frac{d p_{1}}{2 \pi} \frac{1}{q^{2}+p_{1}^{2}+m^{2}} \ldots \int_{-\infty}^{\infty} \frac{d p_{n}}{2 \pi} \frac{1}{q^{2}+p_{n}^{2}+m^{2}} \\
& \times \int_{-\infty}^{\infty} d z_{1} \ldots d z_{n} t\left(p_{n} ; z_{n}, z_{1}\right) \sigma_{\|}^{*}\left(z_{1}\right) t\left(p_{n} ; z_{1}, z_{2}\right) \sigma_{\|}^{*}\left(z_{2}\right) \ldots t\left(p_{n} ; z_{n-1}, z_{n}\right) \sigma_{\|}^{*}\left(z_{n}\right),
\end{aligned}
$$

where

$$
t\left(p ; z, z^{\prime}\right)=\sum_{\ell=0,1} \cos \left(z p+\frac{\ell \pi}{2}\right) \cos \left(z^{\prime} p+\frac{\ell \pi}{2}\right) .
$$

This expression simplifies dramatically when we assume a $\delta$-function background

$$
\sigma_{\|}^{*}(z)=\delta(z-L)+\delta(z+L)
$$

The integrals over $z_{i}$ not only become not only trivial, but identical, thus

$$
\begin{aligned}
\mathcal{E}_{3 \mathrm{D}}^{(n)}\left(\sigma_{\|}^{*}, \Lambda\right) & =-\frac{(-2 \lambda)^{n}}{2 n} \int^{\Lambda} \frac{d^{3} q}{(2 \pi)^{3}} \sum_{\ell=0,1}\left[\int_{-\infty}^{\infty} \frac{d p}{2 \pi} \frac{\cos \left(p L+\frac{\ell \pi}{2}\right)}{q^{2}+p^{2}+m^{2}}\right]^{2} \\
& =-\frac{1}{2 n} \int^{\Lambda} \frac{d^{3} q}{(2 \pi)^{3}}\left[\frac{-\lambda}{2 \omega(q)}\right]^{n}\left\{\left(1+\mathrm{e}^{-2 \omega(q) L}\right)^{n}+\left(1-\mathrm{e}^{-2 \omega(q) L}\right)^{n}\right\}
\end{aligned}
$$

where the cutoff $\Lambda$ appears as an argument for $\mathcal{E}$ to remind us that this is a regulated Casimir energy. We would like to mention that there is no physical interpretation of this cutoff because it cannot be directly linked to either the renormalization scale or the Fourier spectrum of the background field. This cutoff merely serves to render eq. (39) finite for all $n$. Simple power counting indicates that the $n=1,2$, and 3 contributions diverge as $\Lambda \rightarrow \infty$, while, as expected, the contributions of order $n=4$ and higher are finite.

Keeping the cutoff fixed, all orders are finite and can be summed,

$$
\begin{aligned}
\mathcal{E}_{3 \mathrm{D}}\left(\sigma_{\|}^{*}, \Lambda\right) & =\frac{1}{2} \int^{\Lambda} \frac{d^{3} q}{(2 \pi)^{3}} \ln \left[1+\frac{\lambda}{\omega(q)}+\frac{\lambda^{2}}{4 \omega^{2}(q)}\left(1-\mathrm{e}^{-4 \omega(q) L}\right)\right] \\
& =\int_{m}^{\sqrt{\Lambda^{2}+m^{2}}} \frac{d t}{(2 \pi)^{2}} t \sqrt{t^{2}-m^{2}} \ln \left[1+\frac{\lambda}{t}+\frac{\lambda^{2}}{4 t^{2}}\left(1-\mathrm{e}^{-4 t L}\right)\right] .
\end{aligned}
$$


It is easy to verify that the divergences of $\mathcal{E}_{3 \mathrm{D}}\left(\sigma_{\|}^{*}, \Lambda\right)$ as $\Lambda \rightarrow \infty$ correspond to those we analyzed diagram by diagram earlier. However the divergences are $L$-independent, so that we can extract a force per unit area (pressure) between the two plates,

$$
\mathcal{P}_{3 \mathrm{D}}\left(\sigma_{\|}^{*}, \Lambda\right)=-\frac{1}{2} \frac{\partial \mathcal{E}_{3 \mathrm{D}}\left(\sigma_{\|}^{*}, \Lambda\right)}{\partial L}=-\frac{\lambda^{2}}{8 \pi^{2}} \int_{m}^{\sqrt{\Lambda^{2}+m^{2}}} d t \frac{\sqrt{t^{2}-m^{2}} \mathrm{e}^{-4 t L}}{1+\frac{\lambda}{t}+\frac{\lambda^{2}}{4 t^{2}}\left(1-\mathrm{e}^{-4 t L}\right)} .
$$

which is finite as $\Lambda \rightarrow \infty$. Furthermore, it remains finite in the strong limit in which $\lambda \rightarrow \infty$ and the situation reduces to two Dirichlet plates. We then obtain the established result for the force between two Dirichlet plates as a function of $m$ and $L[24$. In the Appendix we show that the same result is obtained in the framework of the interface formalism of Ref. [12].

Next we consider the analogous calculation for a sharp background with spherical symmetry, $\sigma_{\circ}^{*}(\mathbf{x})=\delta(r-R) / 4 \pi R^{2}$. The starting point is the spherical decomposition of the free Green's function

$$
G_{0}\left(x, x^{\prime}\right)=\frac{2}{\pi} \int_{-\infty}^{\infty} \frac{d \omega}{2 \pi} \mathrm{e}^{i \omega\left(x_{0}-x_{0}^{\prime}\right)} \sum_{\ell m} \int_{0}^{\infty} d k k^{2} j_{\ell}(k r) j_{\ell}\left(k r^{\prime}\right) \frac{Y_{\ell m}^{*}(\hat{x}) Y_{\ell m}\left(\hat{x}^{\prime}\right)}{\omega^{2}-k^{2}-m^{2}+i \eta},
$$

where $j_{\ell}$ are spherical Bessel functions and $Y_{\ell m}$ are spherical harmonics. We substitute this decomposition into the expression for the $n$-point function, eq. (35). Again the integral over the time and angular coordinates is trivial. It can be factorized to extract the $n$-th order contribution to the energy $E_{3 \mathrm{D}}^{(n)}\left(\sigma_{\circ}^{*}\right)$,

$$
\begin{aligned}
E_{3 \mathrm{D}}^{(n)}\left(\sigma_{\circ}^{*}\right)= & \frac{1}{n}\left(\frac{-2 \lambda}{\pi}\right)^{n} \int_{-\infty}^{\infty} \frac{d \omega}{2 \pi} \sum_{\ell}(2 \ell+1) \int_{0}^{\infty} \frac{k_{1}^{2} d k_{1}}{\omega^{2}+k_{1}^{2}+m^{2}} \ldots \int_{0}^{\infty} \frac{k_{n}^{2} d k_{n}}{\omega^{2}+k_{n}^{2}+m^{2}} \\
& \times \int_{0}^{\infty} d r_{1} r_{1}^{2} j_{\ell}\left(k_{n} r_{1}\right) \sigma_{\circ}^{*}\left(r_{1}\right) j_{\ell}\left(k_{1} r_{1}\right) \ldots \int_{0}^{\infty} d r_{n} r_{n}^{2} j_{\ell}\left(k_{n-1} r_{n}\right) \sigma_{\circ}^{*}\left(r_{n}\right) j_{\ell}\left(k_{n} r_{n}\right)
\end{aligned}
$$

where the frequency integral has been been Wick-rotated. As in the case of parallel plates, the contributions for $n=1,2$, and3 diverge. They should be regarded as regulated by a cutoff to be introduced explicitly below.

For the $\delta$-function background the momentum integrals can be done, giving

$$
\begin{aligned}
E_{3 \mathrm{D}}^{(n)}\left(\sigma_{\circ}^{*}\right) & =\frac{1}{n}\left(\frac{-\lambda}{2 \pi^{2}}\right)^{n} \int_{-\Lambda}^{\Lambda} \frac{d \omega}{2 \pi} \sum_{\ell}(2 \ell+1)\left[\int_{0}^{\infty} d k k^{2} \frac{j_{\ell}(k R)^{2}}{\omega^{2}+k^{2}+m^{2}}\right]^{n} \\
& =\frac{2}{n}\left(\frac{-\lambda}{4 \pi R}\right)^{n} \int_{0}^{\Lambda} \frac{d \omega}{2 \pi} \sum_{\ell}(2 \ell+1)\left[I_{\ell+1 / 2}\left(R \sqrt{\omega^{2}+m^{2}}\right) K_{\ell+1 / 2}\left(R \sqrt{\omega^{2}+m^{2}}\right)\right]^{n},
\end{aligned}
$$

where $I_{\nu}$ and $K_{\nu}$ are modified Bessel functions. The sum over $\ell$ is finite for fixed $\omega$, but divergences appear in the $\omega$ integral for small $n$. To regulate these divergences we have introduced a cutoff, $\Lambda$, on the frequency integral. 


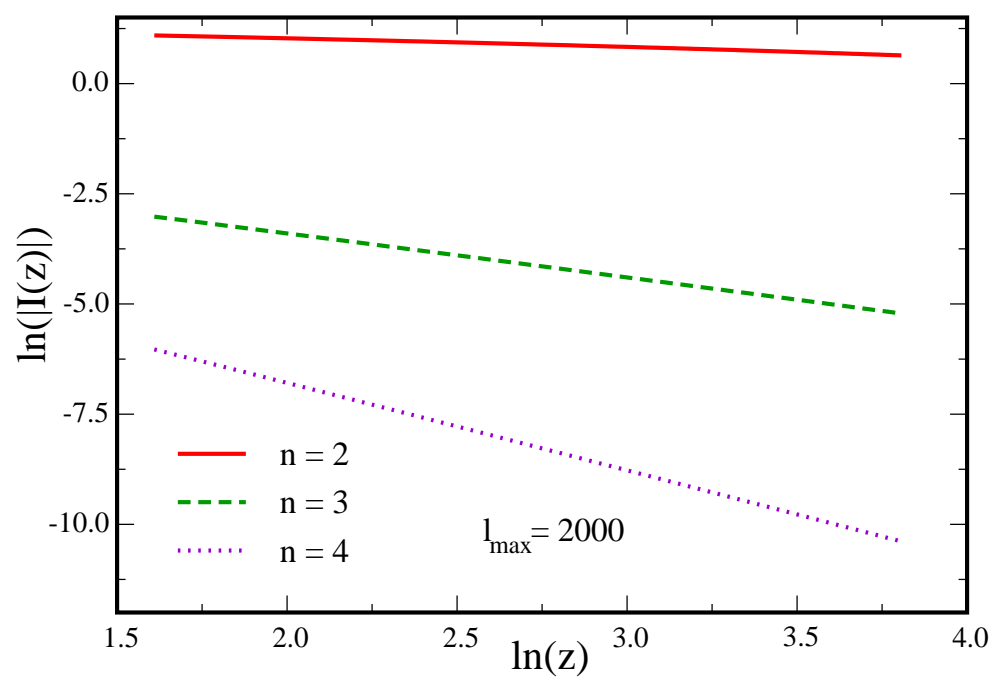

Figure 4: The integrand for $\mathcal{E}^{(n)}$ in eq. (44) as a function of the integral variable $z$ as given in eq. (45). The maximal angular momentum used in this numerical study is $\ell_{\max }=2000$, which is sufficient in the $\omega$ range considered.

The central question is the behavior of the frequency integral after summing over orbital angular momentum $\ell$. For $n=1,2$ we expect divergences because we know that the loop integral in the Feynman graph diverges. Since we did not distinguish between loop and external momenta, Feynman loop integral divergences will show up as divergences in the frequency integral. For $n=1$ we know that $\sum_{\ell}(2 \ell+1) j_{\ell}(z)^{2}=1$. After summing $\ell$, the $k$ integral diverges linearly, as does the $\omega$ integral. These two divergences combine to a quadratically divergent object as expected for the tadpole graph. When we renormalize, the entire $n=1$ term is canceled by a counterterm. In figure 4 we display the integrand,

$$
I_{n}(z)=\sum_{\ell=0}^{\ell_{\max }}(2 \ell+1)\left[I_{\ell+1 / 2}(z) K_{\ell+1 / 2}(z)\right]^{n}, \quad z=R \sqrt{\omega^{2}+m^{2}},
$$

for $n=2,3,4$ in a log-log plot. The sum over $\ell$ is performed numerically up to $\ell_{\max }$ large enough to ensure convergence at the values of $\omega$ considered. This numerical study confirms the results we obtained by studying the $n=2$ and $n=3$ cases in Sections 3 and 4 . For $n=2$ the integrand goes like $\omega^{-\alpha_{2}}$ with $0<\alpha_{2}<1$. The best fit to $\alpha_{2}$ is 0.2064 . So the divergences of the frequency integral is stronger than logarithmic, $i$. $e$. stronger than the loop divergence. For $n=3$ our best fit to $\alpha_{3}$ is 1.0004, consistent with the logarithmic divergence that we expect from the analysis of Section 4. Finally, for $n=4 I_{4}$ behaves like 
$1 / \omega^{2}$ (the best fit to $\alpha_{4}$ is 1.9829 ) yielding a finite frequency integral. So do the higher orders in $n$. This confirms our earlier results that for a $\delta$-function background the singularities are still contained in the lower order Feynman diagrams and are stronger than those from the loop integrals.

Finally, we stress that unlike the case of parallel plates, the divergences in the case of a sphere do depend on the radius of the sphere and therefore afflict the Casimir pressure, making it cutoff dependent.

\section{Conclusions}

We have seen that the Casimir energy of a Dirichlet boundary can be modeled as the limit of the Casimir energy of a background potential, which can then be calculated using the conventional tools of renormalized quantum field theory. Although this energy is finite and unambiguous for any particular smooth background, the limit of this process can still diverge, implying that the energy depends in detail on the properties of the physical material used to impose the boundary conditions. For three space dimensions, the Casimir energy has $\frac{1}{\Delta} \ln \Delta$ divergences both for parallel plates and for the sphere. These divergences should not be confused with the loop divergences of Feynman diagrams: they also appear in diagrams that have no quantum field theory divergences. In particular, the third order diagram needs no renormalization but also diverges in the sharp limit, going like $\ln \Delta$.

This cutoff dependence may or may not enter into physically measurable quantities. The force between rigid bodies is never affected, so our analysis does not alter standard results such as the force between parallel plates. However, it does render meaningless calculations of stresses, such as the Casimir surface tension of the sphere. Such quantities are cutoff dependent, and cannot be defined independently of the material properties that determine the cutoff.

Our results disagree with those of Ref. [18, which also considers low-order Feynman diagrams, but obtains a finite contribution to the stress in the boundary condition limit. We disagree with this calculation on two grounds:

- In Ref. [18] the background fields are analytically continued to fractional dimension. In dimensional regularization, one continues loop momenta to fractional dimensions, but background fields, which are simply complex-valued functions, remain fixed in the physical dimension (see for example [26], p. 662). Analytically continuing the background fields introduces $\sigma$ dependence into the counterterm coefficients $c_{1}$ and $c_{2}$ (see eq. (5)). Counterterm insertions in other diagrams would then render the theory unrenormalizable. 
- In Ref. [18 it is claimed that the contribution of the two-point function to the Casimir energy is finite for $D=3$ space dimensions because the divergences appear in the form $\Gamma(1-D / 2)$, which has poles at $D=2$ and $D=4$ but not $D=3$. However, this is a familiar misinterpretation of dimensional regularization. For example, divergences in QED in $d$ spacetime dimensions appear as $\Gamma(2-d / 2)$, but QED in $4+1$ dimensions is not a finite theory. Similarly, the divergences of $\phi^{4}$ theory come from $\Gamma(1-d / 2)$, but this theory is not finite in $2+1$ or $4+1$ dimensions. The proper prescription in dimensional regularization is to use counterterms to cancel all divergences appearing in dimensions less than or equal to the physical dimension.

If the results described here generalize to the electromagnetic case, as we expect they do, they would invalidate Boyer's result [17] that the conducting sphere experiences a cutoffindependent, repulsive Casimir stress. For such a result to be correct, it is necessary to show that it can be obtained as the limit of an underlying smooth, renormalizable quantum field theory. Otherwise, the Boyer problem cannot be studied without reference to material properties, and the assertion that sphere has repulsive Casimir force is unwarranted.

Acknowledgments We gratefully acknowledge discussions with G. Barton, E. Farhi and K. D. Olum. N. G. is supported in part by the National Science Foundation (NSF) through the Vermont Experimental Program to Stimulate Competitive Research (VT-EPSCoR). R. L. J., V. K., and O. S. are supported in part by the U.S. Department of Energy (D.O.E.) under cooperative research agreement \#DF-FC02-94ER40818. M. Q., O. S. and H. W. are supported by the Deutsche Forschungsgemeinschaft under contracts Qu 137/1-1, Schr 749/1-1 and We 1254/3-2 respectively.

\section{Appendix: Interface Formalism and Force between Plates}

In this section we will verify that the interface formalism of Ref. [12] applied to a $\delta$-function type background gives the standard result for the force [24] between two parallel Dirichlet plates.

The parallel plate geometry is a standard example of an interface problem, where $n$ "trivial" spatial dimensions parallel to the plates are irrelevant for the QFT dynamics and the equations of motion become effectively $m$-dimensional. In the particular case of the parallel plates in three dimensions, $m=1$ and $n=2$.

From the point of view of scattering theory, the interface is characterized by a separation of the wave functions $\Psi(\vec{x}) \sim \psi\left(\vec{x}_{\perp}\right) e^{i \vec{p} \cdot \vec{x}_{\|}}$where $\vec{x}=\vec{x}_{\perp}+\vec{x}_{\|}$, leading to a continuous 
degeneracy of bound and scattering states. The phase shifts $\delta(\vec{k})$ will only depend on the $m$-dimensional momenta $\vec{k}$ in the non-trivial directions, while the integration over the $n$ dimensional trivial momenta $\vec{p}$ leads to an apparent divergence in the total energy. In Ref. [12, it was shown that this spurious divergence is actually absent due to identities in scattering theory that generalize Levinson's theorem 25]. It is then possible to renormalize the Casimir energy per unit area of the interface, $\mathcal{E}=E / A_{\text {plate, }}$, with just the standard field theory counterterms. For the interesting case $m=1, n=2$, the formula for $\mathcal{E}$ reads 25 ]

$$
\begin{aligned}
\mathcal{E} & =-\frac{1}{12 \pi} \sum_{\ell= \pm}\left[\int_{0}^{\infty} \frac{d k}{\pi} h(k) \frac{d}{d k}\left(\delta_{\ell}(k)-\delta_{\ell}^{(1)}(k)-\delta_{\ell}^{(2)}(k)\right)+\sum_{j} h\left(i \kappa_{j, \ell}\right)\right]+\left[\mathcal{E}_{\mathrm{FD}}^{(2)}+\mathcal{E}_{\mathrm{CT}}\right] \\
h(k) & \equiv\left(k^{2}+\mu^{2}\right)^{\frac{3}{2}}-\frac{3}{2} k^{2} \mu-\mu^{3},
\end{aligned}
$$

where $\mu$ is the mass of the fluctuating boson, $\mu^{2}-\kappa_{j, \ell}^{2}=\omega_{j, \ell}^{2}$ are the bound state energies and the counterterm for the first order diagram $\mathcal{E}_{\mathrm{FD}}^{(1)}$ has been chosen to cancel it completely. The specific form of $h(k)$ originates from integrating out the $n$ trivial momenta in dimensional regularization. $\delta_{\ell}(k)$ is the phase shift in the $\ell^{\text {th }}$ partial wave, and $\delta_{\ell}^{(1)}$ and $\delta_{\ell}^{(2)}$ are its first and second Born approximations. Since we are working in three spatial dimensions, a second Born subtraction has to be performed in the phase shift $\delta_{\ell}(k)$, and the corresponding second order diagram $\mathcal{E}_{\mathrm{FD}}^{(2)}$ has to be added back in. This diagram is then renormalized by a standard counterterm $\mathcal{E}_{\mathrm{CT}}$. The final result, eq. (46), yields a finite expression $\mathcal{E}$ for any smooth background $\sigma\left(\vec{x}_{\perp}\right)$.

The phase shifts in eq. (46) are defined through the $m=1$ dimensional problem of a massive boson scattering off a potential $\sigma(x)$ on a line, and $\ell= \pm$ denotes the two parity channels of that problem. We denote the non-trivial coordinate perpendicular to the plates by $x$ and write $\vec{x}=\vec{x}_{\perp}+\vec{x}_{\|}=x \vec{e}_{\perp}+y_{1} \vec{e}_{\|}^{(1)}+y_{2} \vec{e}_{\|}^{(2)}$.

Before we study the sharp limit $\sigma_{\|}(x)=\delta(x-L)+\delta(x+L)$, it is useful to follow [12] and rewrite eq. (46) as an integral over the imaginary axis $k=i t$ using analytic properties of scattering data. This approach combines the scattering and bound state contribution to eq. (46) in a simple and concise formula which is also better from a numerical point of view [12].

The general procedure is explained thoroughly in Ref. [12. Here, it suffices to mention that the phase shift may be expressed in terms of the Jost functions, $2 \delta_{\ell}(k)=i \ln F_{\ell}(k) / F_{\ell}(-k)$. Since the integrand in eq. (46) is odd in $k$, we can extend the integral to $[-\infty, \infty]$ and deform the contour to a large circle in the upper complex $k$-plane, where $F_{\ell}(k)$ is meromorphic. Owing to the two Born subtractions, the integrand falls fast enough at $|k| \rightarrow \infty$. In addition, the residues from simple poles at the bound states $k=i \kappa_{j}$ cancel the explicit bound state contribution in eq. (46), leaving only the discontinuity across the branch cut of the power 
$\left(k^{2}+m^{2}\right)^{\frac{3}{2}}$ in $h(k)$,

$$
\mathcal{E}=\frac{1}{4 \pi^{2}} \int_{m}^{\infty} d t t \sqrt{t^{2}-m^{2}} \sum_{\ell= \pm}\left[\nu_{\ell}(t)-\nu_{\ell}^{(1)}(t)-\nu_{\ell}^{(2)}(t)\right]+\mathcal{E}_{\mathrm{FD}}^{(2)}+\mathcal{E}_{\mathrm{CT}}
$$

where $\nu_{\ell}(t)=\ln F_{\ell}(k=i t)$ is a real function of $t$.

Now we are prepared to study the sharp limit $\sigma(x)=\delta(x+L)+\delta(x-L)$, which we interpreted as a two-channel problem on the half-line $x \geq 0$. Using the well-known Jost functions in the symmetric and antisymmetric channel, respectively,

$$
F_{ \pm}(k)=1+i \frac{\lambda}{2 k}\left[1 \pm e^{2 i k L}\right] \quad \Longrightarrow \quad \nu_{ \pm}(t)=\ln \left[1+\frac{\lambda}{2 t}\left(1 \pm e^{-2 L t}\right)\right]
$$

it is readily seen that the $t$-integral in eq. (47) diverges even at finite coupling $\lambda$. This is the divergence of the Casimir energy in a sharp background from our previous discussion. The $t$-integral in eq. (47) is a compact way of summing all Feynman diagrams of order three and higher. As shown in Section 5 the divergence of this sum in the sharp limit is exclusively due to the third order diagram $\mathcal{E}_{\mathrm{FD}}^{(3)}$. To see this, we subtract one more Born term in eq. (47) and add back in the corresponding Feynman diagram $\mathcal{E}_{\mathrm{FD}}^{(3)}$. Then the $t$-integral (now the sum of all diagrams of order four and higher) will be finite, while the third order diagram (which would be finite for a smooth background) diverges ${ }^{2}$, cf. eq. (39),

$$
\mathcal{E}_{\mathrm{FD}}^{(3)}=\frac{\lambda^{3}}{48 \pi^{2}} \int_{m}^{\infty} d t \frac{\sqrt{t^{2}-m^{2}}}{t^{2}}\left[1+3 e^{-4 L t}\right] .
$$

There is no field theoretic counterterm to renormalize this diagram and thus the divergence of the Casimir energy implies a real physical cutoff dependence.

In the present case, however, this cutoff-dependence has no physical consequences since the logarithmic divergence in eq. (49) comes from the first term in the brackets, which turns out to be independent of the distance $d=2 L$ between the plates. The same holds for the second order diagram $\mathcal{E}_{\mathrm{FD}}^{(2)}$. As a consequence, the physically measurable pressure $\mathcal{P}=-d \mathcal{E} / d(2 L)$ on the plates is finite even though the total Casimir energy diverges. This is again just our main statement about the cutoff independence of the Casimir force between rigid bodies.

After a little algebra, the subsequent strong coupling limit $\lambda \rightarrow \infty$ yields a finite result ${ }^{3}$

\footnotetext{
${ }^{2}$ Note that it is (far) simpler to compute this diagram by (Born) expanding $\nu_{\ell}(t)$ in powers of the coupling $\lambda$ and inserting in eq. (47), than to compute the Feynman diagram directly.

${ }^{3}$ Note that the finite contributions to the force cancel between the Feynman diagram and the corresponding Born subtraction for both the $2^{\text {nd }}$ and $3^{\text {rd }}$ order contributions. This is important since those terms,
} 
for the Casimir pressure between two parallel plates in three dimensions.

$$
P(d)=-\frac{\partial \mathcal{E}}{\partial(2 L)}=-\frac{C(m d)}{d^{4}}, \quad C(s) \equiv \frac{1}{2 \pi^{2}} \int_{s}^{\infty} d \tau \frac{\tau^{2} \sqrt{\tau^{2}-s^{2}}}{e^{2 \tau}-1}
$$

where $d=2 L$ is the distance between the plates. This result can also be derived from eq (41) with $\lambda \rightarrow \infty$ and $\Lambda \rightarrow \infty$. The integral $C(s)$ becomes particularly simple in the massless case, where we recover the standard result

$$
P(d)=-\frac{\pi^{2}}{480 d^{4}}, \quad m=0
$$

\section{References}

[1] S. K. Lamoreaux, Phys. Rev. Lett. 78, 5 (1997).

[2] U. Mohideen and A. Roy, Phys. Rev. Lett. 81, 4549 (1998) arXiv:physics/9805038.

[3] G. Bressi, G. Carugno, R. Onofrio, and G. Ruoso, Phys. Rev. Lett. 88, 041804 (2002) arXiv:quant-ph/0203002.

[4] For a recent review, see M. Bordag, U. Mohideen and V. M. Mostepanenko, Phys. Rept. 353, 1 (2001) arXiv:quant-ph/0106045.

[5] H. B. Casimir and D. Polder, Phys. Rev. 73 (1948) 360.

[6] H. B. G. Casimir, Kon. Ned. Akad. Wetensch. Proc. 51, 793 (1948).

[7] V.M. Mostepanenko and N.N. Trunov, The Casimir Effect and its Application, Clarendon Press, Oxford (1997), K. A. Milton, The Casimir Effect: Physical Manifestations Of Zero-Point Energy, River Edge, USA: World Scientific (2001).

[8] N. Graham, R. L. Jaffe, V. Khemani, M. Quandt, M. Scandurra and H. Weigel, arXiv:hep-th/0207205.

[9] E. Farhi, N. Graham, P. Haagensen and R. L. Jaffe, Phys. Lett. B 427, 334 (1998) arXiv:hep-th/9802015, E. Farhi, N. Graham, R.L. Jaffe, and H. Weigel, Phys. Lett. B 475, 335 (2000) arXiv:hep-th/9912283, Nucl. Phys. B 585, 443 (2000) arXiv:hep-th/0003144, Nucl. Phys. B 630, 241 (2002) arXiv:hep-th/0112217.

which are of order $\lambda^{2}$ and $\lambda^{3}$, respectively, would otherwise prevent us from taking the strong coupling limit $\lambda \rightarrow \infty$. Incidentally, this observation implies that we could have computed the force more easily by making only one Born subtraction and adding nothing back in. 
[10] For a comprehensive review see: N. Graham, R. L. Jaffe and H. Weigel arXiv:hep-th/0201148 in M. Bordag, ed., Proceedings of the Fifth Workshop on Quantum Field Theory Under the Influence of External Conditions, Intl. J. Mod. Phys. A 17 (2002) No. 6 \& 7.

[11] N. Graham, R. L. Jaffe, V. Khemani, M. Quandt, M. Scandurra and H. Weigel, Nucl. Phys. B 645, 49 (2002) arXiv:hep-th/0207120.

[12] N. Graham, R. L. Jaffe, M. Quandt and H. Weigel, Phys. Rev. Lett. 87, 131601 (2001) arXiv:hep-th/0103010.

[13] P. Candelas and D. Deutsch Phys. Rev. D20 (1979) 3063.

[14] P. Candelas, Ann. Phys. 143 (1982) 241.

[15] K. Symanzik, Nucl. Phys. B190 (1981) 1.

[16] L. S. Brown and G. J. Maclay, Phys. Rev. 184, 1272 (1968).

[17] T. H. Boyer, Phys. Rev. 174 (1968) 1764.

[18] K. A. Milton, arXiv:hep-th/0210081, to be published in Physical Review D.

[19] R. L. Jaffe, arXiv:hep-th/0307014.

[20] G. Barton, J. Phys. A 34, 4083 (2001).

[21] L.-H. Chan, Phys. Rev. D55 (1997) 6223.

[22] G.V. Dunne, Phys. Lett. B467 (1999) 238.

[23] J. Baacke, Z. Phys. C53 (1992) 402; J. Baacke and A. Sürig, Z. Phys. C73 (1997) 369

[24] M. Bordag, D. Hennig and D. Robaschik, J. Phys. A 25 (1992) 4483.

[25] N. Graham, R. L. Jaffe, M. Quandt and H. Weigel, Annals Phys. 293 (2001) 240 arXiv:quant-ph/0104136.

[26] M. E. Peskin and D. V. Schroeder, "An Introduction to Quantum Field Theory", Perseus Book Publishing, Cambridge, Ma. (1995). 